\title{
Hand-assisted laparoscopic donor nephrectomy: a single centre experience
}

\author{
Tomasz Jakimowicz ${ }^{1}$, Michał Macech ${ }^{1}$, Amro Alsharabi ${ }^{1}$, tukasz Romanowski ${ }^{1}$, Tadeusz Grochowiecki ${ }^{1}$, \\ Dorota Lewandowska ${ }^{2}$, Piotr Kaliciński ${ }^{3}$, Magdalena Durlik², Leszek Pączek ${ }^{4}$, Sławomir Nazarewski ${ }^{1}$ \\ ${ }^{1}$ Department of General, Vascular and Transplant Surgery, Medical University of Warsaw, Warsaw, Poland \\ 2Department of Transplantation Medicine and Nephrology, Warsaw Medical University, Warsaw, Poland \\ ${ }^{3}$ Department of Children's Surgery and Organ Transplantation, Children's Memorial Health Institute, Warsaw, Poland \\ ${ }^{4}$ Department of Immunology, Transplantology and Internal Diseases, Medical University of Warsaw, Warsaw, Poland
}

Videosurgery Miniinv 2016; 11 (4): 283-287 DOI: https://doi.org/10.5114/wiitm.2016.64997

\begin{abstract}
Introduction: The advantages of a minimally invasive nephrectomy are a faster recovery and better quality of life for the donors. Until recently, the majority of donor nephrectomies in Poland were done by open surgery.

Aim: To present a single centre experience in hand-assisted laparoscopic donor nephrectomy (HALDN).

Material and methods: The first videoscopic left donor nephrectomy in Poland was performed in our department in 2003 using a hand-assisted retroperitoneal approach. From 2011, we changed the method to a transperitoneal approach and started to harvest also right kidneys. Since then, it has become the method of choice for donor nephrectomy and has been performed in 59 cases. Preoperatively, kidneys were assessed by scintigraphy and by angio-computed tomography. We harvested 32 left and 27 right kidneys. There were double renal arteries in 2 cases and triple renal arteries in 1 case. The warm ischaemia time (WIT) was 80-420 s (average $176.13 \mathrm{~s}$ ); operative time was 85-210 min (average $140 \mathrm{~min}$ ). Results: All procedures were uncomplicated, and all donors were discharged after 2-8 days with normal creatinine levels. The average follow-up period lasted 23 months (1-51 months). Out of all of the cases, 1 case had two minor complications, while all others were uneventful. None of the donors were lost to follow-up. All of the kidneys were transplanted. There were 2 cases of delayed graft function (DGF) and 2 cases of ureter necrosis. One of those kidneys was lost in the third postoperative week.

Conclusions: Our limited experience shows that HALDN is a safe method and should be used routinely instead of open surgery.
\end{abstract}

Key words: kidney transplantation, videoscopic nephrectomy, hand-assisted laparoscopy, kidney donation.

\section{Introduction}

Although there are attempts to increase the quantity of potential kidneys for transplantations, significant shortages of available organs are still observed. The quality of the harvested grafts is crucial for the benefit of recipients. A living donor nephrectomy for a kidney transplant is a valuable option.
Using kidneys harvested from a living donor compared with kidneys obtained from a deceased person proves to be a better option due to graft survival rates, convincing cost-effectiveness and improved quality of life for the recipient [1].

Open living nephrectomy had been the standard procedure performed for kidney procurement during the last decades. Although a number of studies pre-

\section{Address for correspondence}

Tomasz Jakimowicz MD, PhD, Department of General, Vascular and Transplant Surgery, Medical University of Warsaw, 1a Banacha St,

02-097 Warsaw, Poland, phone: +48 2259924 67, fax: +48 2259924 68, e-mail: tomj@wum.edu.pl 
sented very low perioperative mortality and morbidity, there was still a need for a low invasive technique to provide shorter recovery. Then, after the first case described by Ratner et al. in 1995 [2], various methods of mini-invasive donor nephrectomy met the above-mentioned expectations. Therefore, videoscopic methods became the gold standard for donor nephrectomy, because of the advantages of a faster recovery, shorter hospital stay and better quality of life for the donors. In addition, these methods help potential donors decide to donate, which in turn expands the donor pool $[3,4]$.

In the United States in 2009, over $95 \%$ of donor nephrectomies were done by minimally invasive techniques [5]; however, in Europe, only $66 \%$ of donor nephrectomies were performed in this way, with a huge gap between eastern and western Europe [6]. Poland is one of the countries where the rate of use of a minimally invasive technique is low and should be improved. Until recently, the majority of donor nephrectomies in Poland were performed by open surgery. Moreover, a very small percentage of the kidneys are obtained from living donors. In

Table I. Patient characteristics

\begin{tabular}{|lc|}
\hline $\begin{array}{l}\text { Type of previous surgery or } \\
\text { concomitant disease }\end{array}$ & $\begin{array}{c}\text { Number } \\
\text { of donors }\end{array}$ \\
\hline Ipsilateral adrenalectomy & 1 \\
\hline Cholecystectomy (laparoscopic) & $4(3)$ \\
\hline Appendectomy & 10 \\
\hline Caesarean section & 11 \\
\hline Hysterectomy & 4 \\
\hline Ovarian cystectomy & 1 \\
\hline Subtotal thyroidectomy & 1 \\
\hline ESWL & 1 \\
\hline Fibroids & 2 \\
\hline Osteoarthritis & 1 \\
\hline Nephrolithiasis & 1 \\
\hline Hypercholesterolaemia & 1 \\
\hline Cholelithiasis & 1 \\
\hline Liver haemangiomas & 1 \\
\hline Hyperthyroidism & 1 \\
\hline Psoriasis & 1 \\
\hline Nutcracker syndrome & 1 \\
\hline Hypertension & 3 \\
\hline
\end{tabular}

2014, 1121 kidneys were transplanted, and only 55 of them were from living donors [7].

\section{Aim}

The objective of this study was to present a single centre experience in hand-assisted laparoscopic donor nephrectomy (HALDN).

\section{Material and methods}

The first videoscopic donor nephrectomy in Poland was done in the Department of General, Vascular and Transplant Surgery on 18 June 2003 using a hand-assisted retroperitoneal approach. The next two cases used the same approach [8]. The 3 cases involved left kidneys. From 6 June 2011, we changed the method to a transperitoneal approach and started to harvest right kidneys using a hand-assisted laparoscopic method. Since then, this method has become the method of choice for donor nephrectomy in our department and has been used in 59 cases. Since the first HALDN was performed in the department, there have been only 7 open donor nephrectomies. The reasons for open surgery in those 7 cases were as follows: decision of the donor (4 cases), necessity of simultaneous open treatment of median arcuate ligament syndrome (1 case) and multiple renal arteries (considered contraindications for laparoscopic surgery at the beginning of our experience, one left and one right kidney - 2 cases). As our experience improved, we have not performed open nephrectomies for kidney transplantations since March 2013; however, all patients were informed about the possible risks and benefits of both open and laparoscopic approaches.

A donor preoperative assessment was performed by scintigraphy and angio-computed tomography (angio-CT). If the difference in a kidney's glomerular filtration exceeded $10 \%$ according to scintigraphy, the better-functioning kidney was left to the donor. In a case of equal function, the kidney with the easier anatomy was taken; this is usually the left kidney. We harvested 32 left and 27 right kidneys. There were double renal arteries in 2 cases and triple renal arteries in 1 case - all anastomosed on the bench table to the side of the main artery or into the common trunk.

There were 42 women and 17 males. The average age was 44 years (range: 19-63 years). All donors were Caucasians. Donor characteristics are presented in Table I. Twenty-five (42\%) donors had previous 
abdominal surgery. In total, 31 (52.5\%) donors had at least one disease or underwent another operation. One donor had a previous ipsilateral adrenalectomy performed in our department due to an unclear lesion found on angio-CT. A histopathological examination revealed an adenoma. Subsequently, the patient underwent HALDN 6 weeks later.

A patient was positioned on the right side for left kidney procurement and on the left side for the right procurement. For the right kidney, a 6-7-cm Pfannenstiel or lower abdominal midline incision was performed according to previous procedures or per the patient. An upper abdominal $6-\mathrm{cm}$ midline incision was performed for left kidney procurement. Hand assistance was achieved using Gelport (Applied Medical, Rancho Santa Margarita, CA, USA). Two 15-mm trocars were placed, one for the camera and one for the harmonic scalpel or endostapler. For the first five right kidney procurements, additional trocars for liver retraction were also used mainly due to lack of experience. After dissection of the kidney from the peritoneum, Gerota's fascia and retroperitoneal fat (using a harmonic scalpel and left hand assistance), the ureter was closed by 3-4 clips and cut proximally. Renal vessels were closed separately with endostaplers (Endo-GIA, Covidien, Mansfield, Minneapolis, USA). The artery was cut first followed by the vein. The kidney was removed manually through an Alexis wound retractor (Applied Medical, Rancho Santa Margarita, CA, USA) and placed into cold $\left(4^{\circ} \mathrm{C}\right)$ Ringer solution. After removing the staple line from the vessels (requires 2-mm shortening of the artery and vein), cold perfusion with Belzer UW Cold Storage Solution (Columbia, South Carolina, USA) was performed. Additionally, we infused heparin until the venous outflow was clear. After that, the kidney was placed in a cold UW solution. After haemostasis control, the wounds were closed. In selected cases, an abdominal drain was left for $24 \mathrm{~h}$.

Adult transplantations (30 cases) were performed in our department, and a recipient's surgery started directly after assessment of the removed kidney. Paediatric transplantations (29 cases) were performed in the Department of Paediatric Surgery and Transplantation of the Children's Memorial Hospital in Warsaw, so the beginning of that type of surgery was at kidney arrival (about 20 min of transport). Kidney transplantations were performed using a standard technique with preference for hypogastric artery anastomosis in adult recipients.
The donor follow-up period consisted of round visits at the first and third months after the HALDN and then annually. Morphology, biochemical and creatinine levels were assessed, and urine tested for microalbuminuria.

\section{Results}

All procedures were uneventful for the donors, and they were discharged after 2-8 days with normal creatinine levels. In 1 case involving a right kidney, an arterial branch was unintentionally cut intraoperatively and was successfully re-anastomosed on the bench table.

The warm ischaemic time (measured from the first artery clamping to the beginning of cold perfusion) was 80-420 s (average: $176.13 \mathrm{~s}$ ). The skinto-skin operative time ranged from 85 to $210 \mathrm{~min}$ (average: $140 \mathrm{~min}$ ).

The average follow-up period of 23 months (1-51 months) was uneventful in all but 1 case, and no donors were lost to follow-up. One (1.69\%) patient had contamination of the wound after discharge; the patient was readmitted and treated conservatively with vacuum and antibiotic therapies. This patient's complication occurred at the hand-port midline incision. The wound did not require debridement, and it closed spontaneously (Grade 1, Clavien scale) [9]. Unfortunately, the same patient subsequently had a Clostridium difficile infection and was readmitted on the $14^{\text {th }}$ day after the operation (Grade 2a, Clavien scale).

None of the donors had a creatinine level above $1.5 \mathrm{mg} / \mathrm{dl}$, and no microalbuminuria was observed.

All kidneys were transplanted. There were 2 cases of transient DGF (3.38\%), one in a highly sensitised recipient with a second kidney transplantation. Moreover, the patient had antiphospholipid syndrome, and the first kidney was lost in the early postoperative period due to thrombosis. Therefore, the patient received a high dose of low molecular weight heparin perioperatively, developed a haematoma on the $1^{\text {st }}$ post-transplant day and required another operation. The second DGF was in a kidney with a predonation undetected renal artery branch aneurysm. After an uncomplicated HALDN, we discovered a $5-\mathrm{mm}$ aneurysm of the $2-\mathrm{mm}$ branch of the upper pole during cold perfusion. After resection and arterial anastomosis, the kidney was transplanted, but the upper pole seemed to be ischaemic, so 
we re-anastomosed the branch. Finally, the kidney revascularised properly, and after 12 days of DGF, the recipient left the hospital with a stable creatinine level of $2 \mathrm{mg} / \mathrm{dl}$.

Another patient was operated on the $15^{\text {th }}$ postoperative day. We found ureter necrosis and performed anastomosis between the ureter and renal pelvis. Due to persistent urine leakage and signs of infection, the patient underwent another operation 2 days later. There was severe pelvic necrosis, so we did not perform a reconstruction and performed graftectomy. The remaining recipients were discharged with good kidney function.

\section{Discussion}

There are various methods of videoscopic donor nephrectomy, such as laparoscopic and retroperitoneoscopic, hand-assisted, "pure" laparoscopic and robotic-assisted or even techniques through natural orifices (NOTES). According to Yuan's et al. metaanalysis [10], there is no significant difference between the different nephrectomy techniques, and all minimally invasive techniques are as safe as open surgery. However, the benefits of videoscopic methods include a shorter hospital stay, a shorter time to return to work and less intraoperative estimated blood loss without increasing intraoperative and postoperative donor complications or compromising recipient graft function favours. Therefore, we have chosen HALDN as the simplest of all minimally invasive methods. Hand assistance gives maximum safety and can provide compression in extensive bleeding, which was proved by Kokkinos et al. in a meta-analysis [11]. They compared two representative groups that underwent classic laparoscopic or hand-assisted live donor nephrectomies. The investigators found that during HALDN, intraoperative blood loss was significantly smaller, warm ischaemia was shorter and total operation time was shorter. Although conversion to an open procedure occurred in $2.97 \%$ in the hand-assisted group and $4.6 \%$ in the full laparoscopic group, statistical significance was not found. Conversions in the full laparoscopic group were seen mostly due to vessel injury and uncontrollable bleeding up to $1200 \mathrm{ml}$ after staple failure [12]. In our study, no bleeding requiring any blood transfusion was observed.

Additionally, the sense of feeling allows surgeons to easily identify various structures, such as arteries and the ureter. Also, a hand in the abdomen enables surgeons to dissect tissues and to gently retract tissues. This can be time-consuming during a fully laparoscopic procedure. Furthermore, the skin incision for hand assistance is no longer than an incision for kidney removal in a laparoscopic bag during "pure" laparoscopic surgery, and less trocar incisions are needed. Similar observations were made by Kercher et al. [13]. Based on this paper, they found that warm ischaemic times were 2-3 times longer in cases of a full laparoscopic donor nephrectomy compared with HALDN. Øyen et al. found that obese donors have a higher risk of intraoperative bleeding and bowel perforation than patients with a lower body mass index (BMI). The investigators suggested that a videoscopic donor nephrectomy can only be performed in patients with a low BMI [14]. Although the amount of our data is relatively small, we must disagree with the former authors. Similar to Heimbach et al. [15], we found no difficulties or prolonged operation times in cases of higher BMI values. However, our patients had widespread BMI values up to $35 \mathrm{~kg} / \mathrm{m}^{2}$.

The only technical problem for a laparoscopic approach could be the adhesions after previous procedures; however, we operated on nine patients after previous abdominal surgery, and a nephrectomy was still possible even shortly after an ipsilateral adrenalectomy.

The procedure we have chosen requires a transperitoneal approach, which creates the risk of bowel injury or adhesive bowel obstruction. To avoid these risks, a retroperitoneal hand-assisted laparoscopic nephrectomy has been proposed. The randomised trial of Dols et al. [16] enrolled nearly 200 donors for laparoscopic transperitoneal and hand-assisted retroperitoneal kidney nephrectomies. They did not confirm superiority of any method, nor any statistical significance of complications. In our study, we did not observe bowel injury or obstruction during the follow-up period. Theoretically, the risk of adhesions causing bowel obstruction still remains in the longterm follow-up period.

In our series, we had no conversion of what corresponds with a low risk of such adverse events falling from $3 \%$ to $0 \%$ in Nakajima's experience in 700 cases [17]. One serious kidney complication, which resulted in graft loss, was ureter necrosis. The ureter blood supply comes from different sources, so this complication can occur after any method of nephrectomy. However, excessive traction could promote this 
kind of complication. In our case, there was also pelvic necrosis, so the reason for the complication was due to blood supply, not the method of nephrectomy. Some authors also reported ex vivo procedures before transplantation - varying from simple vascular reconstruction to complex procedures of pyelotomy with laser lithotripsy due to staghorn stone [18]. In our group thanks to very good donor selection, apart from arterial reconstruction no other ex vivo procedures were necessary.

\section{Conclusions}

Our limited experience shows that HALDN is a safe method and can be used routinely instead of open surgery. It is necessary to promote living donor nephrectomies for kidney transplantations to keep up with Western countries.

\section{Acknowledgments}

The authors thank Mr. Titus Augustine from Manchester Royal Infirmary for his kind support and assistance in the first cases.

\section{Conflict of interest}

The authors declare no conflict of interest.

\section{References}

1. US Department of Health and Human Services. 2008 Annual Report of the US Organ Procurement and Transplantation Network and the Scientific Registry of Transplant Recipients: Transplant Data 1998-2007.

2. Ratner LE, Ciseck LJ, Moore RG, et al. Laparoscopic live donor nephrectomy. Transplantation 1995; 60: 1047-9.

3. Ratner, LE, Montgomery RA, Kavoussi LR. Laparoscopic live donor nephrectomy: the four year Johns Hopkins University experience. Nephrol Dial Transplant 1999; 14: 2090-3.

4. Schweitzer EJ, Wilson J, Jacobs S, et al. Increased rates of donation with laparoscopic donor nephrectomy. Ann Surg 2000; 232: 392-400.

5. US. Renal Data System (2011). USRDS 2011 Annual data Report: Atlas of end-stage renal disease in the Unites States.

6. Lennerling A, Lovén C, Dobbels F, et al. Living Organ Donation in Europe-Clinical Praxis. In: The EULOD Project Living Organ Donation in Europe Results and Recommendations. Ambagtsheer A, Weimar W (eds). Pabst Science Publishers, Lengerich 2013; 9-26.

7. Available from: http://www.poltransplant.pl/Download/biuletyn2015_www.pdf.

8. Nazarewski S, Gałązka Z, Grochowiecki T, et al. Hand-assisted videoscopic live donor nephrectomy. Pol Przegl Chir 2004; 76: 305-14.
9. Kocak B, Koffron AJ, Baker TB, et al. Proposed classification of complications after live donor nephrectomy. Urology 2006; 67: 927-31.

10. Yuan H, Liu L, Zheng S, et al. The safety and efficacy of laparoscopic donor nephrectomy for renal transplantation: an updated meta-analysis. Transplant Proc 2013; 45: 65-76.

11. Kokkinos C, Nanidis T, Antcliffe D, et al. Comparison of laparoscopic versus hand-assisted live donor nephrectomy. Transplantation 2007; 83: 41-7.

12. Chan D, Bishoff JT, Ratner L, et al. Endovascular gastrointestinal stapler device malfunction during laparoscopic nephrectomy: early recognition and management. J Urol 2000; 164: 319-21.

13. Kercher K, Dahl D, Harland R, et al. Hand-assisted laparoscopic donor nephrectomy minimizes warm ischemia. Urology 2001; 58: 152-5.

14. Øyen O, Andersen M, Mathisen L, et al. Laparoscopic versus open living-donor nephrectomy: experiences from a prospective, randomized, single-center study focusing on donor safety. Transplantation 2005; 79: 1236-40.

15. Heimbach JK, Taler SJ, Prieto M, et al. Obesity in living kidney donors: clinical characteristics and outcomes in the era of laparoscopic donor nephrectomy. Am J Transplant 2005; 5: 1057-64.

16. Dols LF, Kok NF, d'Ancona FC, et al. Randomized controlled trial comparing hand-assisted retroperitoneoscopic versus standard laparoscopic donor nephrectomy. Transplantation 2014; 97: 161-7.

17. Nakajima I, Iwadoh K, Koyama I, et al. Nine-year experience of 700 hand-assisted laparoscopic donor nephrectomies in Japan. Clin Transplant 2012; 26: 797-807.

18. Janczak D, Bolanowska B, Jankowski P, et al. Ex vivo pyelotomy, nephroscopy and holmium laser lithotripsy of a staghorn stone in a donor kidney prior to renal transplant. Videosurgery Miniinv 2015; 10: 286-9.

Received: 22.02.2016, accepted: 12.12.2016. 